\title{
Risk factors for bowel necrosis in patients with hepatic portal venous gas
}

\author{
Hiroyuki Koami · Tsutomu Isa • Tomonari Ishimine • \\ Shinichiro Kameyama · Toshinobu Matsumura • \\ Kosuke Chris Yamada $\cdot$ Yuichiro Sakamoto
}

Received: 28 August 2012/ Accepted: 23 January 2014/Published online: 1 June 2014

(C) The Author(s) 2014. This article is published with open access at Springerlink.com

\begin{abstract}
Purpose To evaluate the risk factors for bowel necrosis in adult patients with hepatic portal venous gas (HPVG).

Methods This retrospective study comprised 33 adult patients treated for HPVG between August, 2008 and December, 2011. The patients were divided into a necrotic group $(n=14)$ and a non-necrotic group $(n=19)$. We analyzed the clinical demographics, laboratory data, multi-detector computed tomography findings, treatments, and outcomes in each group.

Results Abdominal pain, peritoneal signs, systolic blood pressure, aspartate aminotransferase, alanine aminotransferase, lactate dehydrogenase (LDH), small intestinal dilatation, poor enhancement of the bowel wall, and intestinal pneumatosis were all significantly associated with bowel necrosis. Moreover, there were significantly more operative cases and deaths in the necrotic group. Multivariate analysis revealed that systolic BP $(p=0.048)$, LDH $(p=0.022)$, and intestinal pneumatosis $(p=0.038)$ were independent risk factors for bowel necrosis. Thus, we created new diagnostic criteria for bowel necrosis based on these three factors, the sensitivity, specificity, and accuracy of which were $100,78.9$, and $87.9 \%$, respectively.

Conclusions This study demonstrates new and important findings to evaluate the risk factors for bowel necrosis.
\end{abstract}

H. Koami ( $\varangle) \cdot$ T. Isa · T. Ishimine · S. Kameyama ·

T. Matsumura

Department of Surgery, Center for Gastroenterology, Urasoe

General Hospital, Iso 4-16-1, Urasoe 901-2132, Japan

e-mail: amicopter@gmail.com

K. C. Yamada $\cdot$ Y. Sakamoto

Department of Emergency and Critical Care Medicine, Faculty of Medicine, Saga University, Nabeshima 5-1-1, Saga 849-8501, Japan
Using our diagnostic criteria, the indications for emergency laparotomy can be established more accurately.

Keywords Hepatic portal venous gas - Bowel necrosis · MDCT $\cdot$ Logistic regression analysis - Diagnostic criteria

\section{Introduction}

Hepatic portal venous gas (HPVG) was initially described in 1955, in neonates with necrotizing enterocolitis [1]. In 1978, Liebman et al. [2] reported that gas in the portal vein was associated with a mortality rate of $75 \%$. For half a century, HPVG has been considered a poor prognostic factor and an absolute indication for emergency laparotomy [3]. However, a cumulative review of 182 cases of HPVG in adults revealed $38 \%$ mortality in those treated surgically and $39 \%$ in those treated conservatively, without a significant difference in mortality between the groups [4]. In recent years, there have been many case reports of milder disease courses. Faberman et al. [5] reported a mortality rate of only $29 \%$ in 17 patients with portal venous gas seen on computed tomography (CT) and pointed out that HPVG is itself not a predictor of mortality. However, few studies have reported the relationship between HPVG and disease severity. The purpose of our study was to demonstrate the risk factors for bowel necrosis in patients with HPVG.

\section{Materials and methods}

Patient data

This retrospective study included all abdominal multidetector CT (MDCT) scans obtained at one institution, 
Fig. 1 Multi-detector computed tomography (MDCT) findings of the patients with hepatic portal venous gas (HPVG)

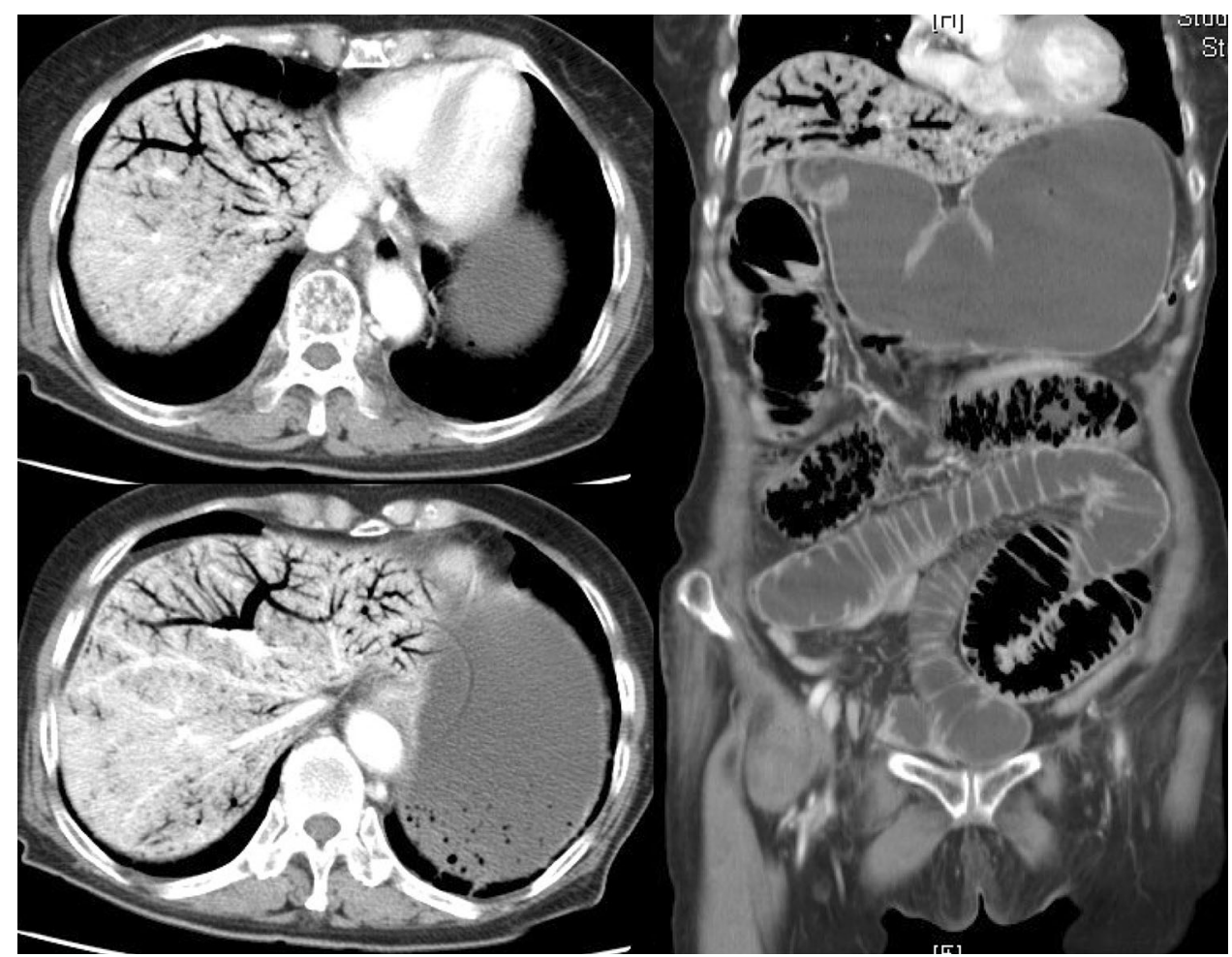

Urasoe General Hospital, between August, 2008 and December, 2011 (Fig. 1). We reviewed the data of 69 patients with HPVG evident on MDCT, retrieved from a computer search. Thirty-six of the 69 patients were excluded from this study because their scans were performed to detect the causes of cardiopulmonary arrest. The remaining 33 patients were divided into two groups based on the presence of bowel necrosis or ischemia: a necrotic group $(n=14)$ and a non-necrotic group ( $n=19$; Fig. 2$)$. We established the presence of bowel necrosis according to the pathological reports and surgical findings. On the other hand, in patients who did not undergo surgery, the bowel necrosis was diagnosed based on the interpretation of radiologists, as we described previously. We analyzed the clinical demographics, including age, sex, admission, abdominal pain, vomiting, peritoneal signs, shock, systolic blood pressure (BP), heart rate, body temperature, and respiratory rate; laboratory data, including white blood cell count (WBC), c-reactive protein (CRP), pH, base excess (BE), total-bilirubin (T-Bil), aspartate aminotransferase (AST), alanine aminotransferase (ALT), creatine kinase (CK), and lactate dehydrogenase (LDH); MDCT findings, including ascites, free air, gastroduodenal dilatation, small intestinal dilatation, large intestinal dilatation, poor enhancement of the bowel wall, intestinal pneumatosis, mesenteric pneumatosis, and gas in the portal vein; diagnoses; treatments; and outcomes of the patients in each group.

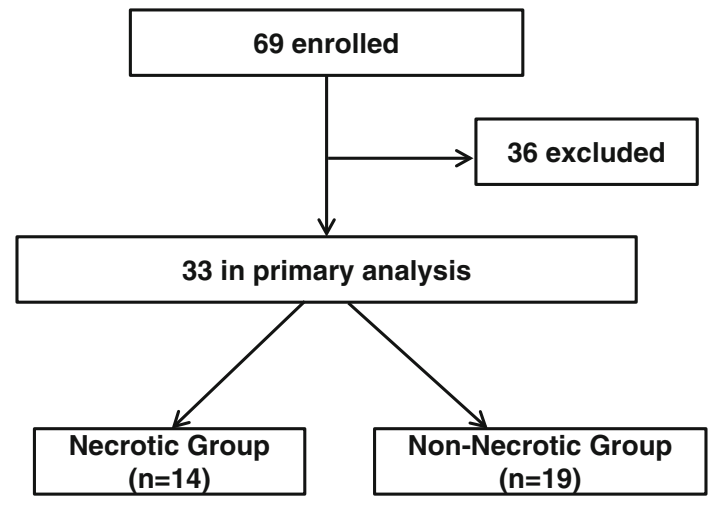

Fig. 2 Study design. Thirty-three patients were divided into two groups based on the presence of bowel necrosis/ischemia

Statistical analysis

To compare differences between the necrotic and nonnecrotic groups, Student's $t$ test, the Mann-Whitney $U$ test, the Chi square test, or Fisher's exact test were used as applicable. The factors with significant differences in the univariate analysis were evaluated in a multivariate analysis. We selected the logistic regression analysis (Forward: LR method) for multivariate analysis. Cut-off values were calculated using the factors with significant differences in the multivariate analysis and used to create diagnostic criteria for bowel necrosis. The data were statistically analyzed using SPSS for Windows version 19. 
Table 1 Baseline clinical characteristics

$B P$ Blood pressure, $H R$ heart rate, $B T$ body temperature, $R R$ respiratory rate, $W B C$ white blood cell, $C R P$ c-reactive protein, $B E$ base excess, $T$-Bil total-bilirubin, $A S T$ aspartate aminotransferase, $A L T$ alanine aminotransferase, $C K$ creatine kinase, $L D H$ lactate dehydrogenase, $M D C T$ multidetector CT

+ Mean \pm standard deviation ( $t$ test)

* Median \pm interquartile range (Mann-Whitney test)

\begin{tabular}{|c|c|c|c|}
\hline & $\begin{array}{l}\text { Necrotic group } \\
(n=14)\end{array}$ & $\begin{array}{l}\text { Non-necrotic group } \\
(n=19)\end{array}$ & $\begin{array}{l}P \text { value } \\
(p<0.05)\end{array}$ \\
\hline \multicolumn{4}{|l|}{ Clinical demographics } \\
\hline Age & $73.8 \pm 9.2$ & $77.4 \pm 12.1$ & $0.355^{\dagger}$ \\
\hline Male & $8(57.1 \%)$ & $11(57.9 \%)$ & 0.966 \\
\hline Admission & $9(64.3 \%)$ & $8(42.1 \%)$ & 0.208 \\
\hline Abdominal pain & $9 / 10(90.0 \%)$ & $4 / 13(30.8 \%)$ & 0.006 \\
\hline Vomit & $8 / 13(61.5 \%)$ & $8(42.1 \%)$ & 0.280 \\
\hline Peritoneal signs & $7 / 13(53.8 \%)$ & $3 / 18(16.7 \%)$ & 0.036 \\
\hline Shock & $8(57.1 \%)$ & $5(26.3 \%)$ & 0.073 \\
\hline Systolic BP (mmHg) & $91.5 \pm 30.3$ & $112.4 \pm 27.3$ & $0.047^{\dagger}$ \\
\hline $\mathrm{HR}(/ \mathrm{min})$ & $117.5 \pm 30.0$ & $103.0 \pm 32.0$ & $0.161^{*}$ \\
\hline $\mathrm{BT}\left({ }^{\circ} \mathrm{C}\right)$ & $36.8 \pm 1.4$ & $36.8 \pm 1.1$ & $0.947^{\dagger}$ \\
\hline $\mathrm{RR}$ (/min) & $24.5 \pm 9.3$ & $24.0 \pm 10.0$ & $0.442^{*}$ \\
\hline \multicolumn{4}{|l|}{ Laboratory data } \\
\hline WBC $(/ \mu \mathrm{L})$ & $15150 \pm 12050$ & $13200 \pm 9400$ & $0.122^{*}$ \\
\hline CRP (mg/dL) & $12.7 \pm 10.8$ & $7.6 \pm 12.2$ & $0.110^{*}$ \\
\hline $\mathrm{pH}$ & $7.37 \pm 0.14$ & $7.40 \pm 0.21$ & $0.781^{*}$ \\
\hline $\mathrm{BE}$ & $-4.2 \pm 8.10$ & $-1.3 \pm 10.18$ & $0.430^{\dagger}$ \\
\hline T-BiL (mg/dL) & $0.7 \pm 1.0$ & $0.6 \pm 0.6$ & $0.567^{*}$ \\
\hline AST (U/L) & $82.5 \pm 289.0$ & $31.0 \pm 24.0$ & $0.012^{*}$ \\
\hline $\operatorname{ALT}(\mathrm{U} / \mathrm{L})$ & $52.5 \pm 68.0$ & $20.0 \pm 22.0$ & $0.038^{*}$ \\
\hline CK (U/L) & $81.0 \pm 833.5$ & $65.0 \pm 56.5$ & $0.286^{*}$ \\
\hline LDH (U/L) & $454.5 \pm 469.5$ & $232.0 \pm 115.0$ & $0.019^{*}$ \\
\hline \multicolumn{4}{|l|}{ MDCT findings } \\
\hline Ascites & $8(57.1 \%)$ & $11(57.9 \%)$ & 0.622 \\
\hline Free air & $0(0.0 \%)$ & $2(10.5 \%)$ & 0.324 \\
\hline Gastroduodenal dilatation & $11(78.6 \%)$ & $10(52.6 \%)$ & 0.126 \\
\hline Small intestinal dilatation & $13(92.9 \%)$ & $11(57.9 \%)$ & 0.030 \\
\hline Large intestinal dilatation & $9(64.3 \%)$ & $11(57.9 \%)$ & 0.710 \\
\hline $\begin{array}{l}\text { Poor enhancement of the bowel } \\
\text { wall }\end{array}$ & $7 / 9(77.8 \%)$ & 2/11 (18.2\%) & 0.012 \\
\hline Intestinal pneumatosis & $13(92.9 \%)$ & $11(57.9 \%)$ & 0.030 \\
\hline Mesenteric pneumatosis & $11(78.6 \%)$ & $9(47.4 \%)$ & 0.070 \\
\hline Gas in the portal vein & $10(71.4 \%)$ & $8(42.1 \%)$ & 0.095 \\
\hline \multicolumn{4}{|l|}{ Treatment and outcome } \\
\hline Operation performed & $8(57.1 \%)$ & $3(15.8 \%)$ & 0.017 \\
\hline Dead & $10(71.4 \%)$ & $7(36.8 \%)$ & 0.049 \\
\hline
\end{tabular}

Data are expressed as the number of patients and ratios (\%) or mean \pm standard deviation (or median \pm quartile deviation). Values of $p<0.05$ were considered significant.

\section{Results}

This study comprised 19 men and 14 women, with a mean age of 76 years (range 51-93 years). Of the 33 patients, 10 had bowel obstruction, 7 had non-occlusive mesenteric ischemia, 4 had ischemic colitis, 3 had supra-mesenteric artery thrombosis, 1 had liver injury, and 8 had other diseases or complications.

Univariate and multivariate analyses

According to univariate analysis, age and male gender distribution was not significantly different in the two groups (Table 1). Among the various parameters examined, abdominal pain $(p=0.006)$, peritoneal signs $(p=0.036)$, systolic $\mathrm{BP}(p=0.047), \mathrm{AST}(p=0.012)$, ALT $(p=0.038)$, LDH $(p=0.019)$, small intestinal 
Table 2 Logistic regression analysis for predicting bowel necrosis

\begin{tabular}{lccrl}
\hline & $\begin{array}{l}\text { Partial } \\
\text { regression } \\
\text { coefficient }\end{array}$ & $P$ value & \multicolumn{1}{l}{ Odds } \\
ratio & $95 \%$ CI \\
\hline Systolic BP & -0.037 & 0.048 & 0.964 & $(0.929-1.000)$ \\
LDH & 0.007 & 0.022 & 1.007 & $(1.001-1.014)$ \\
$\begin{array}{l}\text { Intestinal } \\
\text { pneumatosis }\end{array}$ & 3.632 & 0.038 & 37.793 & $(1.229-1162.062)$ \\
Constant & -1.906 & 0.461 & & \\
\hline
\end{tabular}

$C I$ confidence interval, $B P$ blood pressure, $L D H$ lactate dehydrogenase

dilatation ( $p=0.030)$, poor enhancement of the bowel wall $(p=0.012)$, and intestinal pneumatosis ( $p=0.030)$ were each found to be associated with bowel necrosis (Table 1$)$. There were significantly more operative cases $(p=0.017)$ and deaths $(p=0.049)$ in the necrotic group. All four patients who survived in the necrotic group underwent surgery. Multivariate analysis revealed that systolic BP [odds ratio (OR) 0.964, $95 \%$ confidence interval (CI) 0.929-1.000, $p=0.048]$, LDH (OR 1.007, $95 \%$ CI 1.001-1.014, $p=0.022$ ), and intestinal pneumatosis (OR 37.793, $95 \%$ CI $1.229-1162.062, p=0.038)$ were independent risk factors for bowel necrosis (Table 2).

Calculating the cut-off values and creating the criteria

In the receiver-operating characteristic (ROC) curve analysis of bowel necrosis, the cut-off value of systolic blood pressure was $108.0 \mathrm{mmHg}$, the area under the curve (AUC) was 0.711 , the sensitivity was $57.9 \%$, and the specificity was $78.6 \%$ (Fig. 3a). Furthermore, the cut-off value of LDH was $387.0 \mathrm{U} / \mathrm{L}$, the AUC was 0.748 , the sensitivity was $71.4 \%$, and the specificity was $82.4 \%$ (Fig. 3b). The sensitivity of the presence of intestinal pneumatosis was $54.2 \%$ and the specificity was $88.9 \%$. Next, we examined the number of each of the three factors indicated in the abnormal findings for each patient. All patients in the necrotic group had two or more abnormalities (Fig. 4a). We created diagnostic criteria for bowel necrosis based on three factors; namely, lower systolic BP (108.0 $\mathrm{mmHg}>$ ), higher LDH level (>387.0 U/L), and the presence of intestinal pneumatosis (Fig. 4b). Based on our criteria, bowel necrosis was diagnosed when a patient had more than two abnormal factors. Importantly, our criteria detected necrotic bowel with a sensitivity of $100 \%$, a specificity of $78.9 \%$ and an accuracy of $87.9 \%$.
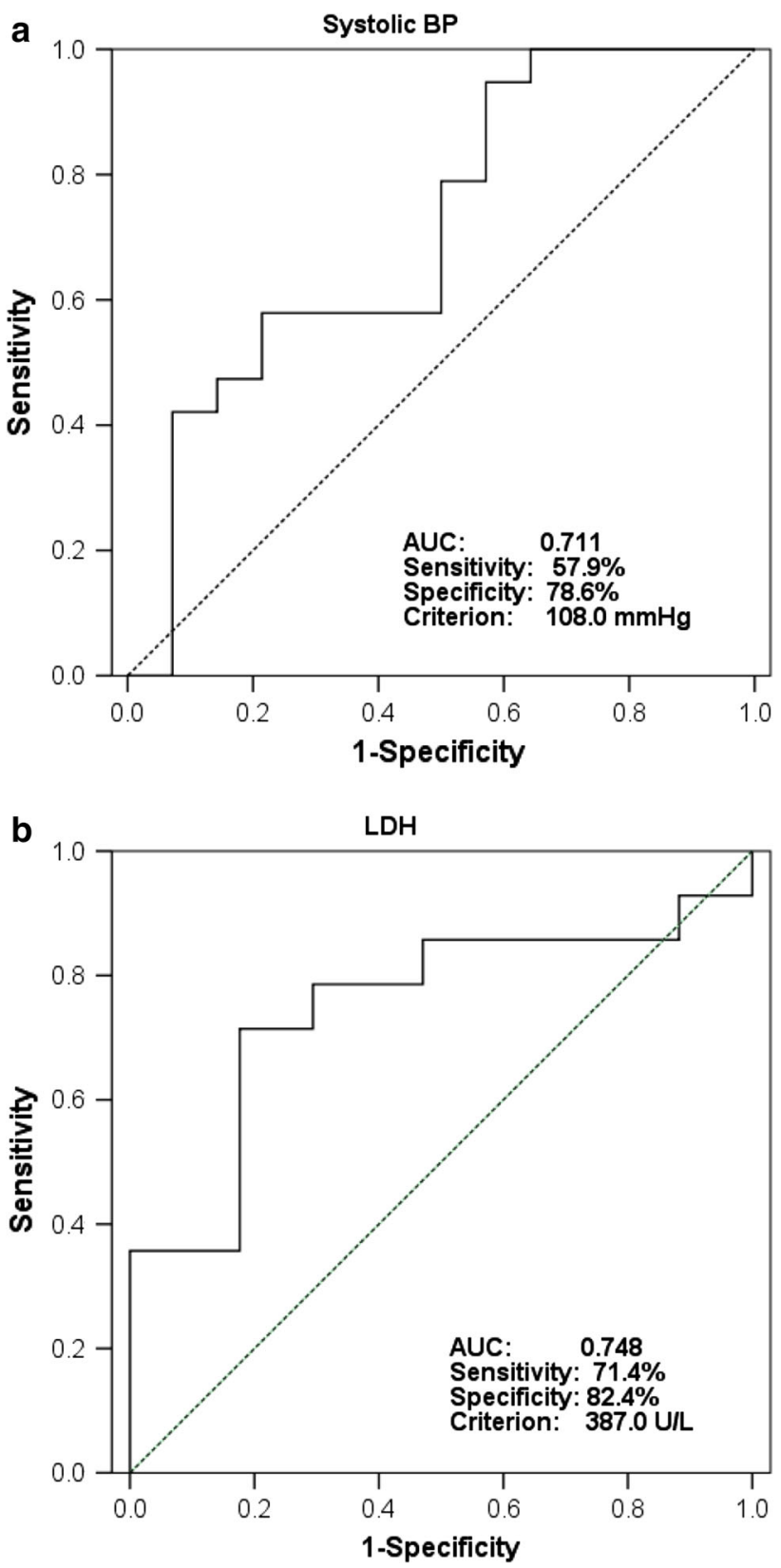

Fig. 3 Receiver-operating characteristic (ROC) curves for detecting bowel necrosis according to systolic blood pressure (BP) (a) and lactate dehydrogenase ( $\mathrm{LDH})(\mathbf{b}) . R O C$ receiver-operating characteristic, $A U C$ area under the curve, $B P$ blood pressure, $L D H$ lactate dehydrogenase

\section{Discussion}

In this study, we identified the risk factors for bowel necrosis in patients with HPVG and created new diagnostic criteria with high sensitivity and accuracy. These criteria consist of three factors that can be easily assessed by physicians in the emergency department and help establish 


\begin{tabular}{c|cc} 
a Number of abnormal parameters & Necrotic Group & Non-necrotic Group \\
\hline 0 & 0 & 3 \\
1 & 0 & 12 \\
2 & 8 & 2 \\
3 & 6 & 2 \\
\hline Total & 14 & 19
\end{tabular}

\begin{tabular}{|c|c|c|}
\hline \multicolumn{3}{|c|}{ Diagnostic Criteria for Bowel Necrosis with HPVG } \\
\hline 1. & \multicolumn{2}{|c|}{ Lower Systolic BP (108.0 mmHg >) } \\
\hline 2. & \multicolumn{2}{|c|}{ Higher LDH level $(>387.0 \mathrm{U} / \mathrm{L})$} \\
\hline 3. & \multicolumn{2}{|c|}{ Presence of Intestinal Pneumatosis } \\
\hline & \multicolumn{2}{|c|}{ number of abnormal parameters } \\
\hline & positive: & 2 or 3 \\
\hline & negative: & 0 or 1 \\
\hline & Sensitivity & $100 \%$ \\
\hline & Specificity & $78.9 \%$ \\
\hline & Positive predictive value & $77.8 \%$ \\
\hline & Negative predictive value & $100 \%$ \\
\hline & Accuracy & $87.9 \%$ \\
\hline
\end{tabular}

Fig. 4 Number of abnormal parameters in the two groups (a). New diagnostic criteria for bowel necrosis in the patients with hepatic portal venous gas (HPVG) (b). According to our criteria, positive bowel necrosis was defined by more than two abnormal findings. The sensitivity was $100 \%$, the specificity was $78.9 \%$ and the accuracy was $87.9 \%$. HPVG hepatic portal venous gas, $B P$ blood pressure, $L D H$ lactate dehydrogenase

whether unstable patients who complain of acute abdominal pain have bowel necrosis.

The number of cases of HPVG treated conservatively has been increasing rapidly; however, few reports have addressed the factors that indicate bowel necrosis and no consensus has been reached. MDCT has become the first choice for HPVG detection and evaluation of the underlying process [6]. CT scans are more sensitive than plain radiographs for depicting small amounts of HPVG [7]. Wiesner et al. [8] reported that contrast-enhanced CT was a powerful investigatory tool to differentiate HPVG with acute mesenteric ischemia from non ischemic pathology.

Reports of intestinal pneumatosis have also been increasing [8-13]. Wiesner et al. [9] stated that band-like pneumatosis and the combination of pneumatosis and portomesenteric venous gas on CT are highly associated with transmural bowel infarction. DuBose et al. [10] conducted a retrospective multicenter study of 500 patients with pneumatosis intestinalis and reported that a lactate value of 2.0 or greater and hypotension/vasopressor use was associated with a predictive probability of $93.2 \%$ of pathologic pneumatosis defined as confirmed transmural ischemia. Moreover, the reported specificities of pneumatosis and portal venous gas for acute bowel ischemia usually approach $100 \%$ [8]. In contrast, according to some reports, intestinal pneumatosis is not useful for diagnosing the severity of HPVG [11]. Furthermore, neither pneumatosis nor portomesenteric venous gas is absolutely specific for transmural bowel wall necrosis in acute bowel ischemia, since the CT findings of both disorders may be observed in patients with only partial mural or even superficial mucosal and submucosal bowel ischemia, which are typically not associated with the same unfavorable clinical outcome [9]. The present study confirmed that intestinal pneumatosis is a significant independent risk factor for bowel necrosis.

Unexpected metabolic acidosis, as well as symptoms such as abdominal pain and peritoneal irritation, is indicative of mesenteric ischemia [6]. Another study suggested that increased lactate levels with anion gaps and/or CT findings suggestive of an ischemic bowel are indications for emergency laparotomy ("aggressive management") [14]. Our findings are not in line with those of the aforementioned reports, which used different modalities to detect HPVG, evaluated a smaller sample size, comprised different articles (such as case reports and reviews), and did not perform a statistical analysis.

The acute physiology and chronic health evaluation (APACHE II) score is designed to measure severity of disease in adult patients admitted to intensive care units. $\mathrm{Wu}$ et al. [15] analyzed data for patients with ischemic bowel-induced HPVG and found that high APACHE II scores and longer length of bowel resection were associated with poor prognosis. To our knowledge, no reports have discussed the relationship between vital signs and bowel necrosis. Although some articles suggest that physical examinations are associated with bowel necrosis [6, 16], our findings did not show a significant correlation between physical examinations and bowel necrosis.

In this study, we created diagnostic criteria based on the three risk factors that were found to be significant independent factors for bowel necrosis. These factors have high sensitivity and accuracy, and can be evaluated easily by physicians in the emergency department. Nowadays, with the development of highly advanced imaging techniques, potentially severe pathologies, such as bowel ischemia, are diagnosed at much earlier stages, allowing prompt treatment and significantly lower mortality [17]. Although it is difficult to diagnose the cause of acute abdominal pain and bowel necrosis in patients with an unstable condition in the emergency department, our new criteria will allow physicians to establish the presence of bowel necrosis and perform surgery as quickly as possible. 
The limitations of our study were that it was retrospective and the study population was small. Moreover, complete surgical or pathological and laboratory evaluations were not available for every patient. However, its findings warrant a study involving a larger sample size in the future. This study demonstrates new and significant findings related to the risk factors for bowel necrosis in patients with HPVG. Using our new diagnostic criteria, the indications for emergency laparotomy can be established more accurately.

Conflict of interest We have no conflicts of interest to disclose.

Open Access This article is distributed under the terms of the Creative Commons Attribution License which permits any use, distribution, and reproduction in any medium, provided the original author(s) and the source are credited.

\section{References}

1. Wolfe JN, Evans WA. Gas in the portal veins of the liver in infants; a roentgenographic demonstration with postmortem anatomical correlation. Am J Roentgenol Radium Ther Nucl Med. 1955;74:486-8.

2. Liebman PR, Patten MT, Manny J, Benfield JR, Hechtman HB. Hepatic-portal venous gas in adults: etiology, pathophysiology and clinical significance. Ann Surg. 1978;187:281-7.

3. Iannitti DA, Gregg SC, Mayo-Smith WW, Tomolonis RJ, Cioffi WG, Pricolo VE. Portal venous gas detected by computed tomography: is surgery imperative? Dig Surg. 2003;20:306-15.

4. Kinoshita H, Shinozaki M, Tanimura H, Umemoto Y, Sakaguchi S, Takifuji K, et al. Clinical features and management of hepatic portal venous gas. Arch Surg. 2001;136:1410-4.

5. Faberman RS, Mayo-Smith WW. Outcome of 17 patients with portal venous gas detected by CT. AJR Am J Roentgenol. 1997;169:1535-8.
6. Hou SK, Chern CH, How CK, Chen JD, Wang LM, Lee CH. Hepatic portal venous gas: clinical significance of computed tomography findings. Am J Emerg Med. 2004;22:214-8.

7. Little AF, Ellis SJ. 'Benign' hepatic portal venous gas. Australas Radiol. 2003;47:309-12.

8. Wiesner W, Khurana B, Ji H, Ros PR. CT of acute bowel ischemia. Radiology. 2003;226:635-50.

9. Wiesner W, Mortele KJ, Glickman JN, Ji H, Ros PR. Pneumatosis intestinalis and portomesenteric venous gas in intestinal ischemia: correlation of CT findings with severity of ischemia and clinical outcome. AJR Am J Roentgenol. 2001;177:1319-23.

10. DuBose JJ, Lissauer M, Maung AA, Piper GL, O'Callaghan TA, Luo-Owen $\mathrm{X}$, et al. Pneumatosis intestinalis predictive evaluation study (PIPES): a multicenter epidemiologic study of the Eastern Association for the Surgery of Trauma. J Trauma Acute Care Surg. 2013;75:15-23.

11. Ho LM, Paulson EK, Thompson WM. Pneumatosis intestinalis in the adult: benign to life-threatening causes. AJR Am J Roentgenol. 2007;188:1604-13.

12. Shimanuki K, Nomura T, Hiramoto Y, Takashima Y, Higuchi K, Sugiyama Y. Pneumatosis Intestinalis in the small bowel of an adult: report of a case. Surg Today. 2001;31:246-9.

13. Imai K, Doi Y, Takata N, Yoshinaka I, Harada K. Successful conservative treatment of pneumatosis Intestinalis associated with intraperitoneal free air: report of a case. Surg Today. 2012;42:992-6.

14. Nelson AL, Millington TM, Sahani D, Chung RT, Bauer C, Hertl $\mathrm{M}$, et al. Hepatic portal venous gas: the ABCs of management. Arch Surg. 2009;144:575-81.

15. Wu JM, Tsai MS, Lin MT, Tien YW, Lin TH. High APACHE II score and long length of bowel resection impair the outcomes in patients with necrotic bowel induced hepatic portal venous gas. BMC Gastroenterol. 2011;11:18-21.

16. Miyoshi O, Shirabe K, Murakami J, Fukuya T, Nakatsuka A, Ayukawa K, et al. Prediction of bowel necrosis in patients with hepatic portal venous gas detected by computed tomography. J Jpn Ass Acute Med. 2009;20:823-8.

17. Abboud B, Hachem JE, Yazbeck T, Doumit C. Hepatic portal venous gas: physiopathology, etiology, prognosis and treatment. World J Gastroenterol. 2009;15:3585-90. 